\title{
Vinculación institucional en red con la emigración altamente calificada. Percepciones de costarricenses en el extranjero
}

\section{Luis Muñoz Varela ${ }^{1}$}

Recepción: 17 de abril de 2014 / Aprobación: 7 de agosto de 2014

\section{Resumen}

Entre diciembre de 2012 y marzo de 2013, en el Instituto de Investigación en Educación (INIE) de la Universidad de Costa Rica, se hizo la aplicación de una pequeña encuesta a 145 personas que en ese momento integraban el registro de la base de datos de la Red TICOTAL ${ }^{2}$, de las cuales se obtuvo respuesta en una cantidad de 73. Este artículo presenta la sistematización de las respuestas obtenidas para uno de los aspectos de la encuesta referido a la percepción que esas personas tienen acerca de las relaciones de intercambio y colaboración que en el país se pueden establecer para desarrollar vinculaciones institucionales en red con ellas. Se hizo también una revisión de artículos y resultados de investigación publicados sobre el tema, sobre todo en el contexto de América Latina. El material bibliográfico consultado sirvió como insumo básico de referencia teórica.

\section{Palabras clave}

"Fuga de cerebros" en Costa Rica; Red TICOTAL; políticas públicas de "repatriación"; emigración altamente calificada; circulación de saberes; redes virtuales de colaboración

\section{Abstract}

From December 2012 to March 2013, at the Instituto de Investigación en Educación (INIE, Spanish Acronym), a small survey was applied to 145 people. At that time, these people were part of the network TICOTAL's database record, from which 73 responses were obtained. This article presents the systematization of the obtained responses for one of the survey's aspects. Such aspect refers to the participants' perception in regards to the development of institutional linkages that the country can establish by creating a network with exchange and collaboration relationships. A review of articles and research results published on this topic, especially in the context of Latin America,

1 Costarricense. Maestro en Ciencias en Investigación Educativa por el Departamento de Investigaciones Educativas (DIE), Centro de Investigación y Estudios Avanzados (CINVESTAV) del Instituto Politécnico Nacional, Ciudad de México. Investigador del Instituto de Investigación en Educación (INIE), Universidad de Costa Rica (UCR). Correo electrónico: luis.munoz@ucr.ac.cr

2 Red de Talento Costarricense en el Extranjero, Academia Nacional de Ciencias. 
was also conducted. The bibliographical material consulted served as basic input of theoretical reference.

\section{Key words}

"Brain drain" in Costa Rica; Network TICOTAL; public policies of "repatriation"; highly skilled emigration; circulation of knowledge; virtual networks of collaboration

\section{Resumo}

Entre dezembro de 2012 para março de 2013, no Instituto de Investigación en Educación (INIE), foi aplicando uma pequena pesquisa para 145 pessoas que, naquela época, eram parte do registro no banco de dados da rede TICOTAL, que obteve-se resposta em um número de 73 . Este artigo apresenta a sistematização das respostas obtidas para um dos aspectos da pesquisa, referindo-se a percepção de que estas pessoas têm relações de troca e colaboração que o país pode ser estabelecido para desenvolver vínculos institucionais em rede com eles. Também foi feita uma revisão de artigos e resultados de pesquisa publicados sobre este tema, especialmente no contexto da América Latina. O material bibliográfico consultado serviu como entrada básica de referencial teórico.

\section{Palavras chave}

"Fuga de cérebros" na Costa Rica; Rede TICOTAL; política pública de "repatriação"; emigração altamente qualificada; circulação do conhecimento; redes colaborativas virtuais

\section{Introducción}

I presente artículo se elabora con base en los resultados de una pequeña encuesta aplicada entre los meses de diciembre de 2012 y marzo de 2013, a una cantidad de 145 personas que en ese entonces integraban el registro de la base de datos de la Red de Talento Costarricense en el Extranjero (Red TICOTAL). Esta Red fue creada en agosto de 2010, por iniciativa de la Academia Nacional de Ciencias de Costa Rica. Cuenta con apoyo del Ministerio de Ciencia y Tecnología (MICIT), el Consejo Nacional para Investigaciones Científicas y Tecnológicas (CONICIT), el Ministerio de Relaciones Exteriores y Culto y de la Asociación Estrategia Siglo XXI. En la página web de la Red se informa lo siguiente:

La Red TICOTAL... es un programa de la Academia Nacional de Ciencias que reúne a científicos e ingenieros costarricenses que estudian o trabajan en el exterior. La red busca conectarlos con el quehacer científico y tecnológico del país mediante el establecimiento de un instrumento permanente de interacción que facilite el intercambio de ideas, contactos y experiencias e identifique oportunidades de cooperación (Red TICOTAL, 2012). 
El trabajo que aquí se presenta consistió en hacer una exploración preliminar en la base de datos de TICOTAL, con la finalidad de conocer cuál es el tipo de información que en ella se registra; cuáles son los indicadores de registro utilizados. La base de datos incluye cuatro áreas disciplinarias: a) Ciencias Naturales y Exactas, b) Ingeniería y Tecnología, c) Ciencias Médicas, d) Ciencias Agrícolas. Por consiguiente, se encuentran excluidas de ella las áreas de Ciencias Sociales, Artes y Humanidades.

A partir de la exploración realizada se decidió elaborar una pequeña encuesta para enviarla por correo electrónico a las personas que en ese momento se hallaban registradas en la base de datos. La encuesta incluyó los siguientes aspectos: a) edad; b) razones para irse a trabajar y a residir en el extranjero; c) tipo de visa que recibieron en el país de destino; d) valoraciones sobre su experiencia de trabajo o de estudio en el extranjero; e) conocimiento que se tiene sobre la existencia de redes académicas y de profesionales costarricenses en los países donde actualmente residen y en Costa Rica; f) relaciones que se mantienen con profesionales y grupos académicos en Costa Rica; g) acciones que considerarían necesarias y pertinentes desarrollar para constituir redes de intercambio de información y para compartir conocimientos entre colegas suyos que se encuentran en Costa Rica y quienes residen en el exterior.

Este artículo sistematiza las respuestas obtenidas en relación con el último punto de la encuesta: "Acciones que considerarían necesarias y pertinentes desarrollar para constituir redes de intercambio de información y para compartir conocimientos entre colegas suyos que se encuentran en Costa Rica y quienes residen en el exterior". Todas las respuestas obtenidas, 73 en total (50,34\% de las personas a quienes se envió la encuesta), han sido debidamente catalogadas en un registro digital específico.

Cabe subrayar que, hasta la fecha de creación de la Red TICOTAL, no se había expresado en el país un interés institucional claro por acercarse a la emigración costarricense altamente calificada. No se había mostrado mayor interés por saber cuál es su situación, dónde se encuentra, qué hace; para promover posibilidades de vinculación dirigidas al desarrollo de proyectos y acciones de colaboración conjuntas con la comunidad científica y profesional en el país.

Las razones de ese desinterés pueden ser de diversa índole: debilidad y desarticulación en los enfoques y las políticas públicas e institucionales en materia de desarrollo de la investigación, la ciencia y la tecnología; insuficiencia de perspectiva acerca del papel que pueden jugar la investigación, la ciencia y la tecnología puestas al servicio de un desarrollo integral del país; carencia de estrategias y mecanismos para dar seguimiento a las personas que se van a estudiar o a trabajar al extranjero; consecuentemente, ausencia de una visión clara acerca de cómo generar condiciones para atraer o revincular con el país a quienes integran la emigración nacional altamente calificada, entre otras. 
La breve encuesta aplicada tuvo por finalidad, desde una preocupación anclada en inquietudes como las anteriores, contribuir a allegar información acerca de lo que opina la emigración costarricense altamente calificada respecto de sus relaciones con el país, en perspectiva de organizar y llevar a cabo iniciativas de colaboración en proyectos y actividades científicas, académicas, de investigación y otras.

\section{Breve referencialidad teórica}

En Costa Rica, en 2006, la Asociación Estrategia Siglo XXI propuso crear un "Programa de repatriación de científicos y tecnólogos costarricenses especialistas en áreas prioritarias para el país, mediante el establecimiento de condiciones apropiadas" (Asociación Estrategia Siglo XXI, 2006, p. 59). A dicha propuesta se agregó, además, la indicación de que en el país se requería contar "con un sistema de incentivos académicos, económicos y técnicos para la reinserción adecuada de investigadores y evitar la fuga de cerebros" (p. 61). Un lustro después, en 2011, el Plan Nacional de Ciencia, Tecnología e Innovación 2011/2014, incluyó la siguiente declaración: "apoyar el establecimiento de una red virtual que vincule a científicos e ingenieros costarricenses residentes en el exterior, para fortalecer el medio científico y productivo nacional (RED TICOTAL)" (Ministerio de Ciencia y Tecnología, 2011, p. 75).

Conforme la así denominada "economía basada en el conocimiento" (Asociación Estrategia Siglo XXI, 2006, p. 23) ha ido incrementándose e instituyendo sus determinaciones en los distintos países del mundo, la movilidad transfronteriza y la emigración de personas con altas calificaciones se hizo también cada vez más intensa, sobre todo desde los países del Sur hacia los del Norte. (CEPAL/SEGIB, 2008, p. 34).

Tal incremento de la migración de personas altamente calificadas se expresa como resultado de "la movilidad de 'cerebros' en el marco de la globalización e internacionalización de los mercados, las llamadas ventajas competitivas y la centralidad del conocimiento en ese proceso" (García de Fanelli, 2008, p. 112). Según señala Balán: "varios autores han subrayado la convergencia de las políticas de apertura de los mercados de trabajo calificado y la competencia internacional por talentos durante las últimas dos décadas" (2009, p. 75).

Otro factor indicado en la investigación especializada lo constituye la emergencia e institucionalización de los sistemas de acreditación de la educación superior. Para el caso de América Latina, a juicio de Didou, dichos sistemas han contribuido a que cada vez se vuelvan "más atractivos y legibles los perfiles de formación de los profesionistas latinoamericanos" (2008, p. 72). Esta situación viene acompañada además de acciones y estrategias emprendidas en los países del Norte, para atraer a las personas de los países del Sur que cuentan con altas calificaciones académicas y profesionales. 
Según observan Balvachevsky y Marques, en el actual contexto de economía globalizada y donde el conocimiento y las capacidades científicas pasan a ocupar un lugar central, los países del Norte intensifican esfuerzos "para atraer mano de obra calificada procedente de otros lugares" (2009, p. 162). De lo que se trata es de captar talento científico y profesional extranjero, que llegue a incorporarse y a fortalecer los propios procesos industriales, dentro de lo cual, en al menos algunos de esos países, también se busca atender una situación existente de desequilibrio entre las demandas de capacidades científicas y tecnológicas y la escasez local de "trabajadores calificados" (Balán, 2009, p. 75).

Las extrapolaciones que hemos hecho a partir de los datos disponibles nos permiten estimar que el número de investigadores e ingenieros originarios de países del Sur que trabajan en el Norte representa más de la tercera parte del número de investigadores en actividad en estos países del Sur, y que el volumen de su producción científica equivale o supera al de las dos terceras partes que permanecen en su país de origen (Schlemmer, 2008, p. 103).

Tal como se sabe, ante las dificultades que afrontan los países del Sur para retener -ya no se diga "repatriar"- a las personas con altas calificaciones científicas y profesionales que emigran, se ha buscado hacer un replanteamiento de la cuestión relacionada con la "fuga de cerebros". Según señala Tejada, ahora urgen "nuevas ideas sobre la definición, manifestación y movilidad internacional del capital humano [que] han orientado diversas perspectivas de estudio y líneas de acción" (2012, p. 69).

El concepto de "fuga de cerebros" apareció en la década de 1950, refiriéndose con él a las repercusiones negativas que acarreaban "los desbalances de los flujos internacionales de la mano de obra altamente calificada entre países menos y más desarrollados" (Didou, 2009, p. 25). Schlemmer (2008) y Vargas (2013) coinciden en indicar, por su parte, que el concepto en cuestión fue inicialmente formulado en Gran Bretaña, en un momento en el que ese país, agobiado por la ruinosa situación económica provocada por la Segunda Guerra Mundial, se hallaba afrontando una importante pérdida de personas altamente calificadas. En una significativa proporción, dichas personas emigraban hacia Estados Unidos y, también, en menor escala, a Canadá.

Pronto, sin embargo, se cayó en la cuenta también de que la "fuga de cerebros" no representaba un acontecimiento que afectara únicamente a Gran Bretaña o a algunos de los países de Europa. Involucraba, de igual manera, una situación que se extendía a escala mundial, propiciando beneficios para los países ricos y pérdidas para los países pobres (Schlemmer, 2008, p. 102).

Años después, en las décadas de 1980 y 1990, el enfoque sobre la "fuga de cerebros" comenzó a variar. De una parte, se tenía por confirmado que la emigración altamente calificada constituía un problema para el cual los países que habían intentado establecer estrategias y mecanismos para retener y repatriar a los "talentos fugados" no habían logrado tener mayor éxito en la aplicación de esas medidas (Tejada y Bolay, 2005, p. 7). 
Durante los últimos años, conforme la globalización económica ha ido instituyendo sus determinaciones a escala planetaria, la "fuga de cerebros" se intensifica, entre otros factores, por la "facilidad de las comunicaciones y transportes internacionales, la convergencia de los niveles de escolarización y la mundialización de los procesos de producción" (Didou, 2009, p. 41). Asimismo, ahora como en el pasado, Estados Unidos continúa siendo el país que recepta la mayor proporción de la migración altamente calificada, aun cuando existen otros países que también han pasado a convertirse en polos importantes de atracción, la mayoría de ellos localizados en la así denominada "zona OCDE" (Didou, 2009, p. 33).

La naturaleza actual de la emigración altamente calificada es compleja y se manifiesta de diversas maneras. Si en el pasado se expresaba como un acontecimiento de carácter unidireccional, que iba desde los países del Sur hacia los del Norte, en la actualidad, la mundialización y la transnacionalización de los procesos de producción provoca que los flujos de la movilidad acontezcan en diferentes direcciones.

Lo importante es tener en cuenta que la emigración altamente calificada, en la actualidad, aun cuando sigue siendo muy alta desde los países del Sur hacia los del Norte y puede preverse que seguirá incrementándose en el transcurso de los próximos años, las condiciones del presente inducen a replantearse las estrategias para atender el problema de pérdida que la "fuga de cerebros" implica especialmente para los países del Sur. Atender esta problemática representa una necesidad y un desafío que ningún país puede soslayar.

En vista de los limitados o nulos alcances que han mostrado tener las estrategias de repatriación, se plantea ahora, por ejemplo, la creación de redes y el establecimiento de vínculos a distancia. La visión de "fuga de cerebros" da paso a nociones tales como la de "diáspora", "revinculación" y "circulación de saberes". Esto se funda en la expectativa de que, más allá de las pérdidas que en efecto la emigración de competencias acarrea para los países, es posible desarrollar otro tipo de acciones que puedan contribuir a revincular las competencias fugadas, por ejemplo, por medio de regresos de corta estancia para participar en proyectos de investigación, pasantías docentes, capacitaciones y otras actividades. Las nuevas perspectivas de abordaje del problema proponen, en consecuencia:

Transformar la emigración (pérdida de personal) por recuperación (de conocimientos). El concepto de "circulación de saberes" pasa a ser clave en este nuevo paradigma, por las oportunidades de nuevas formas de trabajo que facilitan las TICs, como es el surgimiento de redes de conocimientos de múltiples características, cuya efectividad puede ser beneficiosa tanto para los países de destino de la emigración, como para los países de origen (García Guadilla, 2009, p. 2).

Tejada subraya que las personas emigrantes "desarrollan y mantienen vínculos a través de las fronteras... y que cuentan con una identidad propia y un sentido de pertenencia a varios lugares al mismo tiempo" (2012, p. 73). De 
aquí que también se les pueda denominar como "comunidades sin fronteras" (p. 74). Desde esa óptica, antes que concentrar esfuerzos exclusivamente en acciones de "repatriación", de lo que se trataría ahora es de fomentar la transferencia y la circulación de saberes, promover y facilitar la internacionalización de las comunidades científicas nacionales en vinculación en red con sus colegas residentes en el exterior, convocar a la emigración altamente calificada a participar en proyectos nacionales de investigación y desarrollo (Schlemmer, 2008, p. 105). Promover la repatriación sin generar las condiciones propicias para el retorno, no tiene mayor sentido; a menos que eso se haga de una manera muy selectiva, en relación con determinados ámbitos específicos.

Por medio de modalidades y estrategias apropiadas, los países pueden desarrollar acciones para crear relaciones con las personas integrantes de la emigración altamente calificada, de tal manera que eso permita a dichas personas retomar los vínculos con su país de origen y participar en actividades académicas y en proyectos para mejorar las capacidades en investigación, ciencia y tecnología (Tejada y Bolay, 2005, p. 9). Esto puede favorecer la circulación de saberes, en perspectiva de fortalecer las propias capacidades nacionales científicas y de investigación, lo mismo que servir a la vez como un incentivo para asegurar la retención.

Es importante además que los países conozcan cuáles son las políticas de cooperación para el desarrollo que existen en los países de destino de la emigración altamente calificada. Esto requiere, desde luego, la existencia de una clara política de acciones integrada a escala interinstitucional; es decir, la existencia de planes de acción interinstitucional en los que participen las distintas instituciones nacionales relacionadas con la investigación, la ciencia y la tecnología, así como los actores de la sociedad civil y del sector privado.

En Costa Rica, hasta la reciente aparición en 2012 de la Red TICOTAL, la atención institucional dada a la emigración altamente calificada había sido bastante escasa o incluso nula. En cuanto a políticas públicas, es en el último Plan Nacional de Ciencia, Tecnología e Innovación donde aparece la siguiente declaración:

Deben crearse redes de cooperación que permitan la formulación y ejecución compartida de proyectos entre colegas residentes en el exterior y en Costa Rica. Asimismo, otra línea de acción específica la constituye la de apoyar "la participación de investigadores en eventos científicos, así como la organización de dichos eventos en el país" (Ministerio de Ciencia y Tecnología, 2011, p. 75).

La creación de redes científicas, académicas y de profesionales pasa hoy a adquirir un interés cada vez mayor, tanto en el ámbito de las universidades como en el de otras diversas instituciones relacionadas con la investigación, la ciencia y la tecnología. A propósito de la emigración altamente calificada, las redes facilitan dar seguimiento y establecer una comunicación proactiva con las personas que las integran, a la vez que propician la oportunidad de 
que dicha población se mantenga en contacto entre sí y con sus colegas residentes en el país de origen.

"Las redes de revinculación pueden generar situaciones estimulantes para el desarrollo nacional, pero estas condiciones pueden darse en la medida que existan impulsos locales fuertes y que el dinamismo no provenga solamente desde el exterior" (Pellegrino, s.f., p. 14). Ante una situación como la esbozada, la orientación que se propone es la de relevar las propias necesidades de investigación y de generación de conocimientos existentes a escala de las sociedades nacionales, apoyadas en una política pública acorde y en un sistema institucional que le dé su apropiada sustentación.

\section{Metodología}

\section{Encuesta}

Este artículo se elabora con base en información obtenida por medio de la aplicación de una encuesta a 145 personas que en diciembre de 2012 y marzo de 2013 integraban el registro total de la base de datos de la Red TICOTAL de la Academia Nacional de Ciencias de Costa Rica. Los aspectos contenidos en la encuesta fueron ya mencionados en la Introducción.

Las respuestas recibidas fueron 73 en total, las cuales se integraron en un archivo digital. De ese total, se toman aquí en cuenta 62, debido a que 11 de las personas informantes indicaron que ya habían regresado al país. En este artículo se presenta básicamente la sistematización de las respuestas obtenidas con respecto a la última pregunta de la encuesta: ¿Cuáles acciones considera usted que se podrían implementar para constituir redes de intercambio de información y para compartir conocimientos entre colegas suyos que se encuentran en Costa Rica y ustedes que residen y laboran en el extranjero?

\section{Breve descripción de las personas que respondieron la encuesta}

De las 62 personas aquí tomadas en cuenta, su residencia actual en el extranjero presenta una alta concentración en Estados Unidos: 60,7\% de ellas (37) se localizan en ese país. Esta proporción es bastante coincidente con el dato que ofrece Remedi (2009, p. 95) respecto a la emigración altamente calificada de América Latina que tiene a Estados Unidos como país de destino: $60 \%$. Entretanto, una proporción de 6,6\% (4) se encuentra en Alemania, 4,9\% (3) lo están en Canadá y también 4,9\% (3) en España. En Francia y Gran Bretaña se localizan 3,22\% (2) personas en cada país. Otros países en los que se distribuye la residencia del restante 23\% (14) son: Argentina (1), Bélgica (1), Brasil (1), Cuba (1), Francia (2), Gran Bretaña (2), Italia (1), Japón (1), México (1), 34 Países Bajos (1), Portugal (1), Trinidad y Tobago (1). 


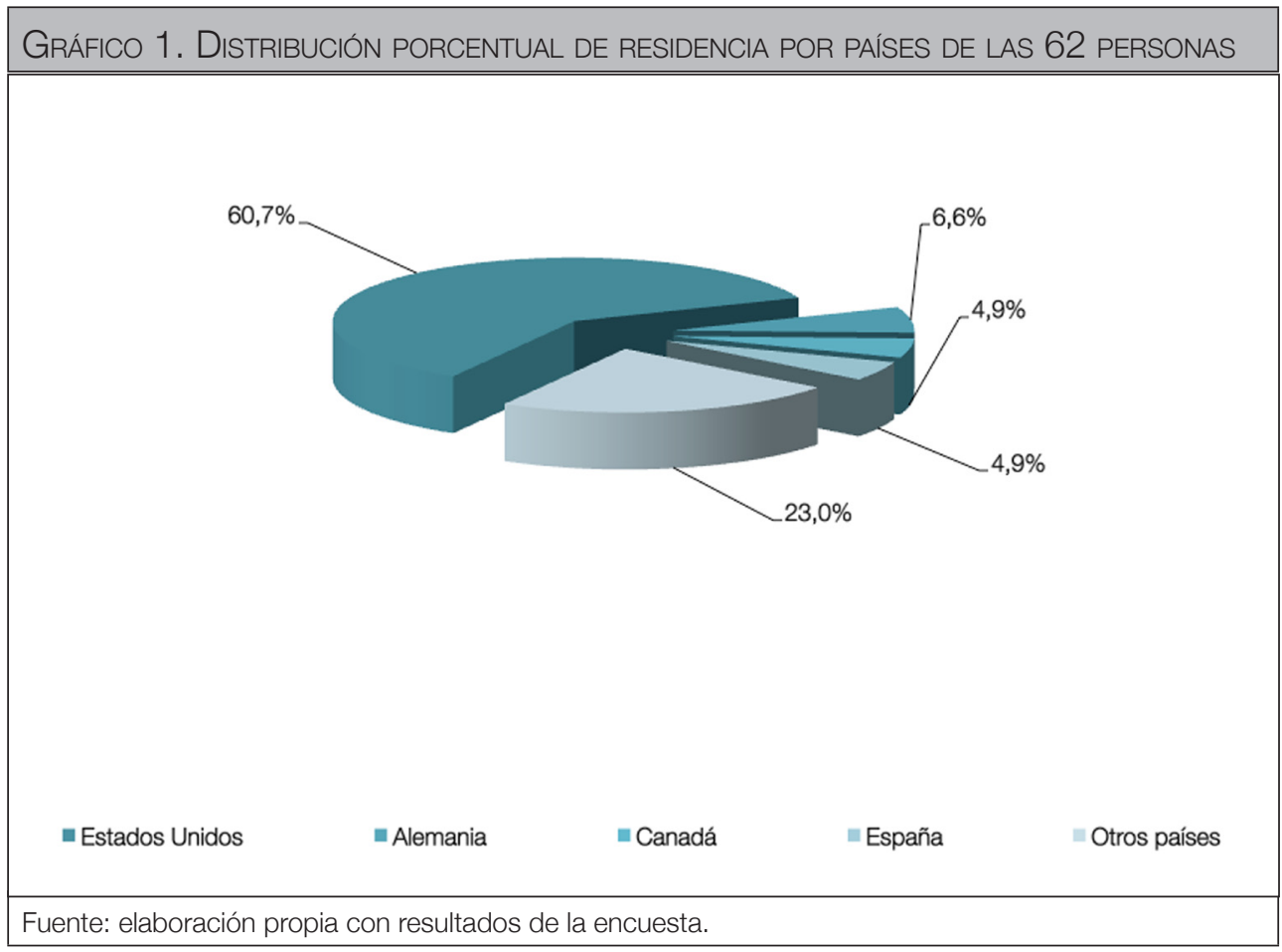

En su lugar, por regiones del mundo, la distribución de residencia actual de las 62 personas es la siguiente: a) 40 personas (67,2\%) se localizan en Norteamérica; b) 15 (24,6\%) en Europa; c) 4 (6,6\%) en América Latina y el Caribe (ALyC) y d) 1 (1,6\%) en Asia. Por su parte, las estimaciones globales de la migración de personas con altas calificaciones ubica una proporción de 85\% en los países de la OCDE (Balán, 2009, p. 77). Si se compara esta cifra con la de la distribución por regiones de las 62 personas aquí consideradas, se puede ver que presenta una proporción un poco mayor a la cifra en cuestión: la suma de las proporciones de distribución en América del Norte y Europa da el resultado de $91,8 \%$.

La distribución por género de las 62 personas presenta una amplia proporción de hombres: 75,80\%. Con respecto a la edad, la mayor proporción de las personas se sitúa en el rango de 30 a 39 años (31=50\%), seguida por el de 40 a 60 años (18=29,03\%). En tercer lugar, aparece el rango de 20 a 29 años (11=17,74\%). Solo 1 de estas 62 personas es mayor de 60 años. 
GRÁFICO 2. DISTRIBUCIÓN PORCENTUAL RESIDENCIA POR REGIONES DE LAS 62 PERSONAS

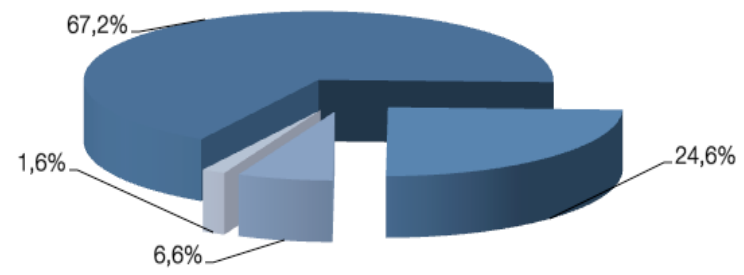

- Norteamérica $\quad$ Europa $\quad$ =ALyC

Fuente: Elaboración propia con resultados de la encuesta.

\section{Sistematización}

Tal como ya se indicó, lo que se ofrece en el presente artículo son los resultados de las respuestas obtenidas para la pregunta acerca de las acciones que podrían ser implementadas para constituir redes de intercambio de información y para compartir conocimientos entre quienes residen en el exterior y sus colegas en Costa Rica. Por ser una pregunta abierta, las respuestas obtenidas refieren a una variedad de temáticas relativamente amplia.

En razón de lo anterior, se procedió a hacer un agrupamiento por temas, de conformidad con las cuestiones planteadas en las respuestas recibidas. Para ello, se tomó en cuenta, en especial, la metodología de sistematización que propone Flick en su libro Introducción a la investigación cualitativa (2012, pp. 192-212). Se obtuvo la siguiente categorización: a) acciones gubernamentales de atención a la emigración nacional muy calificada; b) requerimientos de la política en desarrollo económico, ciencia y tecnología; c) las redes; d) las acciones académicas; e) aprovechamiento de las tecnologías de la información y la comunicación (TIC); f) financiación.

Asimismo, se realizó una exploración bibliográfica lo más amplia posible, en relación con publicaciones realizadas en América Latina en torno a los temas de "fuga de cerebros" y los nuevos enfoques que han venido siendo planteados para abordar dicha cuestión. Con esa bibliografía se construyó un marco teórico buscando establecer una relación estrecha con los temas planteados en las respuestas obtenidas. 


\section{Resultados}

\section{Las acciones gubernamentales de atención a la emigración costarricense altamente calificada}

La percepción expresada por las personas que respondieron la encuesta es que no se conoce, todavía, en Costa Rica la existencia de una política definida, ni tampoco acciones concretas que hayan sido impulsadas por las autoridades de Gobierno, para promover e integrar esfuerzos institucionales de atención y seguimiento a la emigración nacional altamente calificada. Las personas que la integran, se señala, están desvinculadas del país y carecen de espacios que les permita establecer comunicación y buscar la forma de crear relaciones de intercambio y de colaboración con sus colegas en Costa Rica. Quienes respondieron la encuesta, en una proporción de 89\%, mencionan no haber tenido conocimiento, por ejemplo, de alguna iniciativa impulsada para promover y facilitar el desarrollo de intercambios y de colaboraciones en red.

En tal sentido, se plantea en las respuestas obtenidas la necesidad de que el Gobierno de Costa Rica impulse acciones dirigidas a establecer relaciones con las personas integrantes de la emigración altamente calificada. En dicho sentido, una recomendación es la de que el Ministerio de Relaciones Exteriores y Culto (MREC) asuma un papel protagónico, en procura de organizar y llevar a cabo las acciones que se requieren al respecto. Se hace la sugerencia de que las embajadas y las legaciones consulares de Costa Rica pueden hacerse cargo de programar, por ejemplo, encuentros periódicos y pasantías, promoviendo la articulación de espacios y mecanismos de intercambio y de colaboración entre las personas integrantes de la emigración y con sus colegas en Costa Rica.

Se indica también la necesidad de crear y facilitar incentivos y apoyos presupuestarios, que permitan desarrollar la movilidad de las personas interesadas en participar en dichas actividades. Por hallarse situadas en localizaciones geográficas distantes, las personas integrantes de la emigración tendrían que incurrir en hacer largos desplazamientos para poder asistir a esas actividades. En este sentido, la emigración constituye una "diáspora" distribuida en diversos países y regiones del mundo.

Se señala también como importante que el MREC, o bien alguna otra institución nacional, abra y mantenga una página web con incorporación constante de información actualizada, que permita a las personas, tanto las que se encuentran en Costa Rica como las que están en el extranjero, registrarse y establecer vínculos oportunos de comunicación y de intercambio entre ellas.

A propósito de la Red TICOTAL, se señala que su creación ha venido a constituir un primer paso importante. Aun cuando, tal como también se 
indica, parece necesario hacer una mejora en la gestión de la base de datos de la Red, para hacer posible que las comunicaciones sean más flexibles, prontas e interactivas.

La creación de la Red TICOTAL había quedado enunciada en el Plan Nacional de Ciencia, Tecnología e Innovación 2011/2014 del Ministerio de Ciencia y Tecnología (MICIT) donde se le incluyó como una línea de acción dirigida a incrementar "la inversión de los programas de reinserción y atracción de investigadores de alto nivel en centros de investigación y empresas" (Ministerio de Ciencia y Tecnología, 2011, p. 75).

No obstante, en dicho Plan, no llegó a quedar todavía indicada la asignación de una disposición presupuestaria que tuviera por finalidad, por ejemplo, favorecer la movilidad de costarricenses en el extranjero para participar en proyectos $\mathrm{o}$ en encuentros, congresos $u$ otras actividades de naturaleza similar. Se consigna en el Plan la dotación de una suma por US\$720.000, destinada a atraer "al menos 30 investigadores de alto nivel a centros de investigación o empresas" (Ministerio de Ciencia y Tecnología, 2011, p. 75). Con todo, no se especifica cuáles son los términos en que se va a llevar a cabo la contratación y traída al país de esa cantidad de personas. Tampoco se incluye de manera expresa alguna indicación acerca de considerar en la utilización de ese presupuesto a quienes forman parte de la emigración nacional altamente calificada.

\section{Requerimientos de la política pública en desarrollo económico, ciencia y tecnología}

\section{Directrices de la política económica}

En las respuestas aparece la observación de que, en Costa Rica, la orientación de la política económica ha estado centrada en colocar productos de bajo precio en el mercado internacional, lo cual es valorado como una limitación en términos de estímulos al desarrollo de las condiciones y las capacidades nacionales en materia de ciencia y tecnología. Se indica, además, que una situación como esa hace que el país afronte una baja proyección a escala internacional. Tal situación implica que Costa Rica vea disminuidas sus posibilidades para fortalecer las relaciones internacionales de intercambio en el campo de la investigación y la generación de conocimiento científico.

\section{Empresas de base tecnológica}

Se indica que también es importante que la política pública y las instituciones del Estado promuevan la formación de empresas locales de base tecnológica y de investigación, apoyadas con capital nacional y que no dependan de la inversión extranjera directa (IED).

Según se señala, el desarrollo de empresas de base tecnológica puede venir a crear condiciones para dar un mayor realce al perfil de la actividad 
científica y de investigación que se lleva a cabo en el país. A ello se añade la indicación de que una orientación como esta puede venir a representar también un incentivo importante para que las personas que integran la emigración costarricense altamente calificada, se vean atraídas e interesadas a participar en actividades y proyectos de ciencia e investigación en Costa Rica.

La perspectiva propuesta es que la elevación y fortalecimiento de la actividad científica y de investigación contribuye a extender la posibilidad de integrar redes científicas y de investigación, lo que repercutiría en lograr una mejor proyección internacional de la investigación y del quehacer científico que se lleva a cabo en el país, además de que, de esa manera, se puede fomentar una mejora sistemática y continuada de las propias capacidades locales para el desarrollo de la investigación, la ciencia y la tecnología. Esta indicación sugiere, por lo demás, la perspectiva de un modelo nacional de desarrollo basado en la integralidad y en la búsqueda de los equilibrios que contribuyan a potenciar las capacidades científicas y tecnológicas de los diferentes sectores productivos del país.

\section{Regionalización de las actividades científicas y tecnológicas}

Otra acción que se propone en las respuestas obtenidas es la de descentralizar la inversión en ciencia y tecnología, desconcentrándola de la Gran Área Metropolitana Ampliada (GAMA), para llevarla a las diversas localizaciones y regiones geográficas del país. Se plantea, al respecto, que la regionalización puede ayudar a reducir costos de instalación de infraestructura y de operación, a la vez que fomentar un mayor interés por parte de diferentes actores locales (económicos, productivos, institucionales, sociales), en el sentido de desarrollar una gestión más amplia en materia de incorporar la ciencia y la tecnología en las actividades productivas que se llevan a cabo en las distintas regiones geográficas del país.

La incorporación de la ciencia y la tecnología en las actividades productivas y económicas en las regiones, con énfasis en industrias poco desarrolladas, es valorada como de alta importancia, en la medida que ello contribuye a mejorar los rendimientos productivos y económicos, además de que tiene una importante repercusión en términos de generar a las comunidades beneficios de diversa índole: sociales, económicos, educativos, institucionales, culturales.

\section{Capacitación}

Otro tema que aparece en las respuestas y que tiene que ver con la sugerencia de desarrollar empresas de base tecnológica en las regiones es el de la capacitación; una acción en la que pueden participar y colaborar quienes integran la emigración altamente calificada. Se propone que una forma de organizar dichas acciones puede ser por medio de formalizar actividades de capacitación apoyadas con presupuesto gubernamental, para movilizar por temporadas a Costa Rica a las personas que cuenten con trayectorias académicas, científicas y de investigación destacadas. 
Asimismo, se plantea que, en un caso como el de las universidades públicas, estas instituciones podrían organizar programas y acciones de capacitación, en coordinación con las direcciones regionales de los diferentes ministerios que cuentan con oficinas en las distintas regiones del país. Las capacitaciones a desarrollar han de tomar en cuenta la participación de los diversos actores locales, dentro de los cuales es importante incluir también a las poblaciones estudiantiles de la educación media o secundaria.

No obstante, se recalca que siempre va a ser necesario que las acciones que se proponga desarrollar sean definidas desde la propia Costa Rica. Quienes integran la emigración altamente calificada pueden proporcionar una colaboración importante, pero el aprovechamiento de esa colaboración será posible, en la medida en que las iniciativas propuestas formen parte de una política pública definida con claridad y fundada en una visión estratégica del desarrollo nacional, lo mismo que en una adecuada sustentación institucional.

Una acción más que aparece sugerida en las respuestas es la de organizar pasantías en las universidades públicas, aprovechando que todas estas instituciones tienen sedes y recintos distribuidos en diversas localizaciones geográficas del país. La sugerencia esbozada indica que la forma ideal de llevar a cabo tales acciones es por medio del establecimiento de programas articulados de manera interinstitucional, de tal forma que se le pueda dar una sustentación sostenida a las iniciativas que se decida emprender.

Al respecto, cabe mencionar aquí una reciente iniciativa de carácter interinstitucional impulsada en Costa Rica, la cual consiste en el "Proyecto Centro de Capacitación Técnica en Tecnologías Avanzadas". En este proyecto participan de manera conjunta la Asociación Estrategia Siglo XXI, el Instituto Nacional de Aprendizaje (INA) y la Universidad EARTH. Dentro de las finalidades del proyecto figura la de promover la "repatriación" de especialistas con calificaciones de alto nivel, para hacerse cargo del desarrollo de investigación, ciencia y tecnología en ciertas áreas consideradas de interés prioritario. A tal efecto, se propone la creación de un programa de becas, "focalizado en la formación de recurso humano especializado y la atracción de talentos costarricenses destacados en el extranjero" (Ministerio de Ciencia y Tecnología, 2011, p. 33).

\section{La vinculación en red}

De conformidad con la percepción de quienes respondieron la encuesta, el núcleo de sustentación de una red lo otorga precisamente la solidez de pertinencia de una temática específica que la articule y que sea de interés significativo, tanto para la comunidad investigadora involucrada como para otros diferentes actores. Para garantizar la sustentabilidad y asegurar una acción significativa, las relaciones a establecer en red deben estar provistas además de un adecuado balance entre finalidades, organización y cursos de acción. "La gente quiere aprender, compartir, pero también tener impacto en 
un período corto o mediano", señala un joven académico costarricense que labora en una universidad de los Países Bajos.

En el marco de las relaciones e iniciativas a impulsar en red, se señala la necesidad de considerar asimismo una combinación de actividades de encuentro presencial con las que se pueden llevar a cabo por medio de las herramientas y las plataformas de la comunicación virtual. A ello se agrega la indicación de que las acciones deben organizarse con base en una planificación bien definida, que garantice la mayor consistencia posible en su articulación y ejecución y que tenga en cuenta también la compatibilidad de agendas.

Igualmente, señalaron la necesidad de contar con personal capacitado para hacerse cargo de la gestión de la red; personas con conocimiento y experiencia en aspectos de funcionamiento y operación de plataformas virtuales, formulación de planes y proyectos, familiaridad con las temáticas específicas en las que están involucradas las personas participantes en la red. Todo esto hace necesario, además, en razón de dar su sustentabilidad a la red, que se establezca una base oficial de operación en alguna institución determinada.

Se subraya que son muy variadas las acciones que en el marco de una red es posible desarrollar y que pueden aportar beneficios importantes. Al respecto, aparece la sugerencia de que una posible acción puede ser la de organizar proyectos de investigación para llevar a cabo de manera conjunta entre las universidades de Costa Rica y las universidades e instituciones de investigación del extranjero. En lo que concierne a Costa Rica, las comunidades investigadora y estudiantil de las universidades podrían contar con una oportunidad para ampliar las posibilidades de acceso a estancias y capacitaciones en laboratorios muy bien equipados, donde participar en interacción con colectividades científicas y de investigación de frontera altamente consolidadas. Desde el punto de vista de las inversiones financieras, todo esto puede contribuir a paliar en alguna medida la insuficiencia de recursos con la que con frecuencia se ve afectada la investigación en Costa Rica.

Respecto al proceso de constitución de una red, hay también la observación de que, por lo general, se trata de una tarea prolongada, que implica iniciar por el desarrollo de actividades en las que se realice la presentación de propuestas y el intercambio de valoraciones y criterios acerca de la pertinencia y significado de la iniciativa. $Y$ en estas actividades no debiera estar ausente ninguno de los actores que pueda estar interesado en participar en el desarrollo de la iniciativa de que se trate.

Se hace énfasis en que las redes deben ser significativas y promisorias para las finalidades y los intereses de los grupos académicos y de los diversos actores involucrados. Esta es una condición preliminar que debe cumplirse, a fin de que las iniciativas impulsadas se puedan desarrollar de manera adecuada. Asimismo, se indica que no existe mayor posibilidad de llevar a cabo de manera consistente las acciones, que no sea por medio de asegurar una 
dinámica de articulación interinstitucional que promueva el desarrollo constante y continuado de relaciones basadas en agendas temáticas de interés común y compartido. En este sentido, se subraya, que no se debería proponer y lanzar un programa completo, sin antes haber comprobado la magnitud de interés y pertinencia que la temática específica planteada tiene para los diversos actores participantes. En ello, además, está de paso implicada también la posibilidad de asegurar una disposición de financiamiento que le permita a la red desarrollarse y operar.

El desarrollo de interacciones dinámicas y sistémicas en red es considerado como una tarea necesaria por parte de las personas que respondieron la encuesta. Se subraya que, sin lugar a dudas, ese tipo de acciones pueden allegar beneficios importantes para el país, a la vez que constituyen también espacios de interés para la participación y la colaboración de las personas integrantes de la emigración altamente calificada. No obstante, se recalca, a la vez, que hasta el presente, las relaciones que hayan podido establecerse son todavía insuficientes, esporádicas y que no han contado con una organización programática y sistémica que facilite llevar a cabo actividades y proyectos interinstitucionales, lo mismo que para dar espacio al desarrollo de iniciativas de trabajo colaborativo en red.

Al respecto, es importante mencionar que a la pregunta de si se conocía sobre la existencia en Costa Rica de alguna iniciativa de vinculación en red con la emigración altamente calificada, el 59\% de las respuestas obtenidas señala que eso no es así. Asimismo, en una proporción de 53\%, se indica que por propia disposición personal se ha mantenido alguna relación informal con colegas en el país. Además, un $90 \%$ de las personas dice desconocer acerca de la existencia de alguna red de costarricenses en el país donde actualmente residen. El restante 10\% menciona que la única red de cuya existencia en Costa Rica tienen conocimiento es la recién creada Red TICOTAL.

Por último, cabe mencionar que en las respuestas obtenidas, la iniciativa de TICOTAL aparece valorada de manera bastante positiva. Se considera que la base de datos creada por TICOTAL es una herramienta que favorece la comunicación entre las personas que integran la emigración nacional altamente calificada. "Los grupos como TICOTAL son una muy buena idea", señala en su respuesta un estudiante costarricense que realiza sus estudios de doctorado en Italia.

\section{Las acciones académicas}

En la dimensión de las actividades académicas, las respuestas obtenidas hacen énfasis en la importancia que tiene desarrollar iniciativas de intercambio y colaboración, tanto en actividades de investigación como de docencia.

Sin embargo, se esboza la percepción de que, por ejemplo, en el caso 42 de las universidades públicas de Costa Rica, no puede observarse que estas 
instituciones hayan tomado todavía en cuenta en sus programas la importancia de crear y mantener relaciones de intercambio y colaboración con la emigración nacional altamente calificada para coordinar y llevar a cabo actividades académicas y proyectos de investigación.

Según se menciona, son diversas las actividades académicas que pueden ser organizadas y llevadas a cabo con la participación y colaboración de quienes integran la emigración altamente calificada: seminarios, talleres, foros, conferencias. No obstante, de nuevo aquí se hace énfasis en que para ello es necesaria la existencia de una política institucional a la que se le asigne una adecuada disposición presupuestaria. Las relaciones a establecer involucran el desarrollo de un proceso sostenido. Se propone que lo que puede hacerse es programar actividades académicas iniciales y exploratorias, encuentros preliminares donde las personas tengan la oportunidad de intercambiar experiencias y plantear sus intereses y expectativas. Esto con la finalidad de identificar las posibles líneas de trabajo que puedan ser asumidas de manera compartida y colaborativa, para ir construyendo a la vez las respectivas agendas.

En las respuestas se subraya, asimismo, la importancia de contar con una adecuada disposición de infraestructura, equipos y laboratorios, de igual manera que con tiempo asignado a académicas y académicos para que participen en las iniciativas de articulación en red que se puedan impulsar desde Costa Rica.

Asimismo, se propone que una acción que las instituciones de educación superior públicas pueden impulsar es la de establecer programas específicos para promover, facilitar y apoyar la visita de costarricenses que laboran en universidades del extranjero, a fin de que realicen pasantías docentes en las del país durante sus períodos de vacaciones, o bien, para impartir conferencias, participar en proyectos de investigación y en otras diversas actividades. "Yo ya lo hice, pero fue hace como 15 años", menciona una profesora que trabaja desde hace 25 años en una universidad estatal de Estados Unidos. "Fue un curso intensivo y muy bien recibido. Yo sé que en términos burocráticos sí se puede hacer. Solo se tendría que contar con un fondo relativamente modesto para cubrir honorarios básicos".

Se propone también organizar cursos cortos en las universidades del país para las fechas en las que las personas residentes en el exterior están de vacaciones. "Muchos contratos académicos, al menos en las universidades de Estados Unidos, son por nueve meses, en lugar de 12. Esos tres meses de verano podrían ser aprovechados por académicos residentes en el extranjero para realizar proyectos o impartir clases en las universidades públicas de Costa Rica", señala otra profesora que labora en una universidad de Estados Unidos.

Otra acción que se sugiere es la de identificar a académicas y académicos en el exterior que cuentan con una trayectoria destacada y reconocida en investigación, con el fin de que puedan fungir como "líderes de investigación", en laboratorios y unidades de investigación de las universidades y otras 
instituciones del país. La propuesta aquí es la de un programa de "Líderes de Investigación Remotos", que se puede establecer en las distintas áreas disciplinarias. Las personas que asuman la función de líderes mantendrían una comunicación permanente por medios virtuales con sus colegas en el país, a la vez que podrían hacerse cargo de asumir la tutoría de proyectos de estudiantes de doctorado en Costa Rica bajo la modalidad a distancia.

En tal sentido, se indica, además, la posibilidad de que las personas desde el lugar donde se encuentren en el extranjero, puedan asumir la dirección o coordinación de equipos de investigación en Costa Rica, siempre mediante una comunicación que se realice a través de los medios virtuales. Esto podría abonar la ventaja de obviar la cuestión del pago de salarios y otros requerimientos económicos, ya que la persona en el extranjero podría colaborar sin necesidad de ausentarse de la institución donde labora.

Las relaciones y los intercambios académicos deben organizarse tanto de manera virtual como presencial. "Para tratar algunas cuestiones siempre va a ser necesaria la relación presencial, el encuentro cara a cara", señala una de las personas. A este respecto, cabe decir que en algunos países de América Latina, desde hace algunos años ya se han venido desarrollando experiencias en las que se incluyen ambas dimensiones de interacción y encuentro. En el marco de esas experiencias, señala Didou, se "abrieron espacios de reencuentro entre los de afuera y los de adentro, virtuales o presenciales, permanentes o episódicos, en los lugares donde los científicos están asentados o en su país de origen" (2012, p. 95).

\section{Aprovechamiento de las tecnologías de la información y la comunicación (TIC)}

Una cuestión que atrae la atención de las personas encuestadas es la de la utilización de las herramientas y las plataformas de internet para establecer comunidades de comunicación e intercambio. Hasta ahora, según se señala en las respuestas obtenidas, no se ve claro que estas herramientas y plataformas estén siendo utilizadas de la mejor manera en Costa Rica. Es importante hacer uso de ellas, para promover la comunicación, los acercamientos, el intercambio y la creación de redes entre quienes integran la emigración altamente calificada y la comunidad académica e investigadora residente en el país.

Se señala que las tecnologías de la información y la comunicación (TIC) permiten multiplicar y flexibilizar las posibilidades de la comunicación, que ahora pueden hacerse en tiempo real desde diferentes localidades del planeta. El acceso a la información que facilita internet hace posible identificar en qué lugar del mundo se está haciendo investigación sobre las temáticas en las que cada cual está trabajando. Diversas revistas científicas hoy están disponibles en la web, facilitando el acceso a los últimos resultados de investigación y al desarrollo de los intercambios. 
Según se sugiere, las bases de datos y las páginas web de las instituciones públicas de Costa Rica es importante que realicen mejoras y actualizaciones en cuanto al diseño, funcionamiento y contenidos. En relación, por ejemplo, con las universidades públicas, se considera como importante que las páginas web de las unidades académicas y de investigación incorporen información bajo criterios que tomen en cuenta la más amplia divulgación posible de las líneas de investigación en las que se está trabajando.

Lo anterior facilitaría que quienes han emigrado a laborar en universidades e instituciones de investigación del extranjero estén al tanto de la investigación que se está realizando en el país. Esta es una forma de promover que dichas personas se sientan interesadas por ponerse en comunicación con sus colegas en Costa Rica, en búsqueda de establecer relaciones que propicien su participación y colaboración en proyectos y actividades académicas y de investigación.

En el plano de la comunicación que se realiza por medios virtuales, se pueden establecer plataformas de foros de intercambio integradas al sistema normal de correo electrónico. Se indica que este tipo de foros son muy utilizados en la actualidad y que tienen por finalidad la presentación y el intercambio de información en temáticas específicas. Quienes participan pueden colocar sus preguntas, comentarios e información diversa en el foro y todas las personas suscritas la reciben por correo electrónico. De esa manera, el foro pasa a constituirse en un espacio propicio y flexible para la circulación de información, realizar los intercambios y favorecer iniciativas de colaboración en común.

Se señala también en las respuestas, que si se carece de una definición clara de política y de estrategias para desarrollar la vinculación con las personas integrantes de la emigración altamente calificada, será difícil disponer de los indicadores que permitan organizar la información en su presentación vía web y ponerla a disposición de manera adecuada y provechosa. Se recomienda mantener una labor de mejora constante de gestión de las bases de datos virtuales y de las páginas web institucionales, de manera tal que se pueda ofrecer un óptimo acceso e información actualizada con una visión amplia y proactiva de la importancia que tiene dar una visibilidad adecuada y pertinente a la información.

Con respecto a la iniciativa de la Red TICOTAL, la percepción esbozada es la de que, además de desarrollar un registro de profesionales y académicos en el exterior, la base de datos de la Red podría al mismo tiempo abrir un espacio para que quienes en Costa Rica (profesionales, investigadores, académicos) pudieran tener un interés genuino por desarrollar relaciones de intercambio y de colaboración con sus colegas costarricenses residentes en el exterior, también para que se incorporen a la base de datos y participen de manera activa y visible en la Red.

En tal sentido, se propone el desarrollo de una base de datos que ofrezca información sobre las especialidades, intereses y actividades profesionales, 
académicas y científicas de la comunidad investigadora y académica de Costa Rica, para que quienes están en el extranjero puedan tener acceso a dicha información y buscar y proponer posibles relaciones de colaboración.

Sin embargo, se indica que una condición, en particular, cuyo cumplimiento es deseable y que podría aportar significativos beneficios es que las personas que decidan incorporar información lo hagan porque, en efecto, tienen un interés genuino por participar y llevar adelante los intercambios. En la base de datos de TICOTAL: "yo he tratado de colaborar con gente allí, pero cuesta encontrar personas que realmente quieran poner el esfuerzo y que estén entusiasmadas al respecto". Esta es la indicación que hace una profesora e investigadora costarricense con una trayectoria de trabajo de 14 años en una universidad de Estados Unidos. Otra persona señala lo siguiente:

El concepto de TICOTAL es excelente y por eso decidí unirme al grupo. He recibido comunicaciones por parte de ellos en varias ocasiones, pero hasta el momento no de personas que estén trabajando en mi área de investigación en específico. Si TICOTAL tuviera sub-grupos dedicados a temas en particular, o bien si hubieran otras redes similares a TICOTAL para áreas específicas, eso fomentaría ese tipo de intercambio más fácilmente.

La creación de bases de datos con información y registro de la emigración costarricense altamente calificada resulta ser entonces una de las acciones relevantes a desarrollar, pero también debe contarse con una base de datos complementaria o integrada, donde se ofrezca información sobre las comunidades académicas e investigadoras existentes en Costa Rica.

La utilización y gestión de las herramientas y plataformas de internet se propone, asimismo, que ofrezcan acceso abierto de información para las universidades, las empresas, instituciones del Estado, organizaciones gremiales y de la sociedad civil, de manera tal que estas entidades puedan informarse respecto de los diferentes campos y temáticas específicas en las que se encuentran trabajando y haciendo investigación las personas que forman parte de la emigración. A tal efecto, se propone la creación de bases de datos con información específica de universidades, instituciones del Estado, empresas, organizaciones gremiales y de la sociedad civil, que estén interesadas en realizar trabajos de colaboración en el extranjero.

Dentro de otras opciones que se recomiendan está la utilización de las redes sociales disponibles en internet, tales como "Linkedin" o "Facebook". Hoy día, se señala, estas redes son de un uso extendido y facilitan la comunicación y el intercambio de información al interior de grupos temáticos específicos. "Aunque no siempre son confiables las redes virtuales, me parece que resultan muy útiles como herramientas para intercambiar información y para compartir avances y resultados de los proyectos en los que cada cual está trabajando". Esta es la apreciación que facilita un estudiante costarricense que hace sus estudios de doctorado en una universidad de Portugal. 


\section{Financiación}

La existencia y funcionamiento de las redes generan acciones que vienen a propiciar beneficios de importancia para diversas instituciones y actores. Su financiación, por consiguiente, remite a una cuestión que también debe ser asumida de manera conjunta por todas las instituciones que perciban beneficios de las actividades que en el marco de una red se puedan llevar a cabo.

Según se señala en las respuestas obtenidas, una de las principales acciones a desarrollar, a tal efecto, es el diseño de un modelo de gestión para la consecución y la asignación de dicho financiamiento. Como punto de partida, a ello deben contribuir las instituciones del Estado, las universidades, otras instituciones y también las entidades del sector empresarial interesadas.

En todo caso, de lo que se trata es de desarrollar una gestión dirigida de manera específica a la consecución de recursos que permitan proporcionar un soporte sostenido de financiación a las actividades que la red se proponga llevar a cabo. Una dimensión importante y significativa viene a ser también la de la movilidad estudiantil. A este respecto, se sugiere promover y concretar la creación de fondos de investigación para proyectos comunes, donde haya participación de estudiantes y se incluyan líneas de financiación para cursos cortos y pasantías, por ejemplo, de estudiantes costarricenses en las universidades extranjeras donde están trabajando las personas integrantes de la emigración.

Al respecto, es pertinente aquí mencionar que, en el marco del Plan Nacional de Ciencia, Tecnología e Innovación 2011/2013, se incluye la indicación de que con la finalidad de mejorar y fortalecer las capacidades nacionales en materia de "capital natural", ha de procederse a la creación de fondos "para la reinserción de investigadores y apoyo para sabáticos - de extranjeros y de nacionales radicados en el Exterior- así como un apoyo adicional para financiar a las instituciones nacionales que los reciben" (Ministerio de Ciencia y Tecnología, 2011, p. 52).

En las respuestas obtenidas se indica asimismo la importancia de hacer una gestión institucional orientada a la búsqueda de la firma de acuerdos bilaterales entre universidades de Costa Rica y universidades e instituciones del exterior, en particular, con aquellas en las que están las personas integrantes de la emigración altamente calificada. De lo que se trata es de identificar y localizar recursos que puedan facilitar a esas personas su movilidad hacia Costa Rica para participar en actividades académicas y científicas donde haya la oportunidad de interactuar y compartir con la comunidad investigadora y científica nacional, lo mismo que con estudiantes de las universidades del país. 


\section{Consideraciones finales}

De conformidad con las respuestas obtenidas en la encuesta realizada, aun cuando las 62 personas que la contestaron se ubican en espacios académicos y profesionales diversos, la mayoría de ellas manifiesta tener interés y estar en disposición de participar y colaborar en proyectos de investigación y actividades académicas que se puedan llevar a cabo en conjunto con colegas de la comunidad científica e investigadora del país.

El desarrollo de iniciativas al respecto es considerado de importancia, en la medida que quienes integran la emigración altamente calificada pueden proporcionar aportes significativos al país, en las diferentes áreas de la investigación, la ciencia y la tecnología. Las acciones a emprender, sin embargo, deben ser desarrolladas desde Costa Rica y contar con una organización institucional adecuada, sobre todo en aspectos tales como la financiación, la programación de actividades de encuentro para la definición de proyectos a desarrollar en conjunto con instituciones y grupos académicos y de investigación en el país, estrategias y mecanismos de comunicación institucionalizados que favorezcan y aseguren el intercambio flexible y continuo de información (plataformas virtuales interactivas, por ejemplo). La percepción que se esboza al respecto es que, aun cuando ese tipo de acciones pueden llegar a ser de una importancia significativa para el país, hasta ahora, esta no parece haber sido todavía un área de interés claramente definida por las autoridades del Gobierno de Costa Rica, lo mismo que tampoco por las autoridades de otras diversas instituciones del país.

A tal efecto, cabe indicarse que en el Plan Nacional de Ciencia, Tecnología e Innovación 2011/2014 se incluyeron dos menciones a la emigración costarricense altamente calificada, planteándose la necesidad de llevar a cabo acciones que faciliten la repatriación de, al menos, algunas de esas personas, en especial de aquellas que cuentan con formación de alto nivel en las áreas de ciencia y tecnología.

Sin embargo, en Costa Rica, cabe decir que, además, de que es todavía bastante incipiente la preocupación formulada en las políticas públicas por establecer relaciones e impulsar la apertura de espacios de colaboración con participación de las personas integrantes de la emigración nacional altamente calificada, también, es necesario avanzar en dichas políticas hacia una perspectiva que vaya más allá de la de la repatriación. En tal sentido, puede decirse que, en Costa Rica, existe la necesidad de desarrollar un mayor esfuerzo y compromiso institucional por identificar las diversas modalidades y estrategias que puedan instituirse para establecer relaciones de intercambio y colaboración con la emigración nacional altamente calificada.

De conformidad con las respuestas obtenidas, aun cuando la mayoría de las 
en iniciativas de red y colaborar en diversas actividades y proyectos en conjunto con la comunidad científica y académica del país, es importante mencionar que, en una proporción de alrededor de 40\% de ellas, señalan que dentro de sus planes no se encuentra ni un pronto ni un lejano regreso a Costa Rica. Aquí hay, evidentemente, una indicación a la que hay que prestar su debida atención, a propósito de la forma en que debe enfocarse la cuestión y, también, para definir e impulsar las correspondientes estrategias y mecanismos de acción.

Una cuestión importante es que, en Costa Rica, tal como sucede en otros países, a la fecha es deficiente la información y estimaciones que se dispone acerca de cuántas personas puedan formar parte en la actualidad de la emigración costarricense con altas calificaciones académicas y profesionales. Con excepción del registro en desarrollo con que cuenta la base de datos de la Red TICOTAL, no se ha podido identificar en el marco de la presente breve investigación, ninguna otra fuente de información que se haya ocupado en desarrollar un registro similar. Este es uno de los principales pendientes que el país afronta, para lo cual sería necesario empezar por definir los correspondientes indicadores que permita conocer cuál es la situación que presenta Costa Rica en materia de emigración de personas con altas calificaciones científicas y profesionales, así como para identificar cuáles puedan ser las estrategias institucionales que se deben seguir para establecer relaciones de intercambio y colaboración con las personas integrantes de la emigración nacional altamente calificada.

Asimismo, el país requiere contar con políticas públicas e institucionales consistentes en lo que corresponde al desarrollo de la investigación, la ciencia y la tecnología. Es preciso plantearse esas políticas y los correspondientes cursos de acción, desde la perspectiva de la contribución que la investigación, la ciencia y la tecnología pueden proporcionar para apoyar, nutrir y consolidar procesos de desarrollo nacional integral, inclusivo, democratizador y potenciador de las propias condiciones y capacidades productivas del país.

\section{Referencias}

Asociación Estrategia Siglo XXI. (2006). Visión de la ciencia y la tecnología en Costa Rica: una construcción colectiva, Vol. I. San José: Fundación Costa Rica-Estados Unidos de América para la Cooperación. Recuperado de http://www.estrategia.cr/documentos/tomo1.pdf

Balán, Jorge. (2009). Los mercados académicos en el Norte y la migración internacional altamente calificada: el contexto actual de la circulación de cerebros en América Latina. En Sylvie Didou Aupetit y Etienne Gérard (eds.), Fuga de cerebros, movilidad académica y redes científicas. Perspectivas latinoamericanas. Ciudad de México: Instituo Internacional para la Educación Superior en América Latina y el Caribe (IESALC) / Centro de 
Investigación y Estudios Avanzados (CINVESTAV / IRD) /Institut de Recherche pour le Dèveloppment (IRD).

Balbachevsky, Elizabeth y Fabricio Marques. (2009). "Fuga de cerebros" en Brasil: los costos públicos del errado entendimiento de una realidad académica. En Sylvie Didou Aupetit y Etienne Gérard (eds.), Fuga de cerebros, movilidad académica y redes científicas. Perspectivas latinoamericanas. Ciudad de México: IESALC / CINVESTAV / IRD.

CEPAL / SEGIB. (2008). Espacios iberoamericanos. La economía del conocimiento. Santiago de Chile: Naciones Unidas. Recuperado de http://www. cepal.org/publicaciones/xml/9/34459/Espacioliberolll.pdf

Didou Aupetit, Sylvie. (octubre-diciembre, 2008). Presentación. Movilidades académicas y profesionales en América Latina: entre la ignorancia y la polémica. Revista de Educación Superior, XXXVII(148), 71-85.

Didou Aupetit, Sylvie. (2009). ¿Pérdida de cerebros y ganancia de saberes?: la movilidad internacional de recursos humanos altamente calificados en América Latina y el Caribe. En Sylvie Didou Aupetit y Etienne Gérard (eds.), Fuga de cerebros, movilidad académica y redes científicas. Perspectivas latinoamericanas. Ciudad de México: IESALC / CINVESTAV / IRD.

Didou Aupetit, Sylvie. (2012). De una visión retrospectiva a un balance prospectivo: la internacionalización de los sistemas de ciencia y educación superior en América Latina. En Humberto Grimaldo Durán y Francisco López Segrera (comps.), La internacionalización de la educación superior a nivel mundial y regional. Principales tendencias y desafíos. Bogotá: Planeta / Universidad de Colombia, 87-108.

Flick, Uwe. (2012). Introducción a la investigación cualitativa. Madrid: Ediciones Morata y Fundación Paideia Galiza.

García de Fanelli, Ana. (octubre-diciembre, 2008). Políticas públicas frente a la "fuga de cerebros": reflexiones a partir del caso argentino. Revista de Educación Superior, XXXVII(148), 111-121.

García Guadilla, Carmen. (2009). La emigración de personal calificado desde países de América Latina y el Caribe. Perspectiva de IESALC / UNESCO. Caracas: Sistema Económico Latinoamericano y del Caribe. Recuperado de http://www.carmengarciaguadilla.com/articulos/2009_La_Emigracion.pdf

Ministerio de Ciencia y Tecnología. (2011). Plan Nacional de Ciencia, Tecnología e Innovación 2011/2014. San José: Ministerio de Ciencia y Tecnología. 
Pellegrino, Adela. (s.f.). Drenaje, movilidad, circulación: nuevas modalidades de la migración calificada. Recuperado de http://www.google.co.cr/url?sa=t\& $r c t=j \& q=\& e s r c=s \&$ source $=$ web\&cd $=3 \& v e d=0 C D k Q F j A C \& u r l=h t t p \% 3 A \% 2$ F\%2Fwww.cepal.org\%2FCelade\%2Fproyectos\%2Fmigracion\%2FPellegri no.doc\&ei=tnhgUe3NOMTc4AO0j4DgBw\&usg=AFQjCNEBiKUHWdCZ1yd m85nunn3Ss4prwg\&sig2=I5A4-W0Sq7YxCJVW8y22bQ\&bvm=bv.447705 16,d.dmg

Red TICOTAL. (2012). Conozca acerca de TICOTAL. Recuperado de http://ticotal.cr/

Remedi Allione, Eduardo. (2009). Fuga de cerebros y movilidad profesional: ¿vectores de cambio en la educación superior? En Sylvie Didou Aupetit y Etienne Gérard (eds.), Fuga de cerebros, movilidad académica y redes científicas. Perspectivas latinoamericanas. Ciudad de México: IESALC / CINVESTAV / IRD.

Schlemmer, Bernard. (octubre-diciembre, 2008). Migraciones científicas y políticas de cooperación: ¿Qué implicaciones tienen para África? Revista de Educación Superior, XXXVII(148), 123-139.

Tejada Guerrero, Gabriela y Jean-Claude Bolay. (2005). Impulsar el desarrollo a través de la circulación del conocimiento: una mirada distinta a las migraciones de los mexicanos altamente calificados. Ginebra, Suiza: Global Comission on International Migration. Recuperado de http://www.refworld. org/docid/43662f8dd7.html

Tejada Guerrero, Gabriela. (2012). Movilidad, conocimiento y cooperación: las diásporas científicas como agentes de desarrollo. Migración y Desarrollo, 10(18), 67-100. Recuperado de http://infoscience.epfl.ch/record/185224/files/DiasporasCientificas-Tejada.pdf

Vargas Aguilar, Simón. (4 de mayo de 2013). Fuga de cerebros /I. La Jornada. Recuperado de http://www.jornada.unam.mx/2013/05/04/opinion/016a1pol 